\title{
Virtual Force-Field Safety Net for Variable Passive Elastic Leg Joint Limits with a Gait Rehabilitation Robot
}

\author{
Kyle Chisholm, Mojtaba Ahmadi \\ Advanced Biomechatronics and Locomotion Laboratory \\ Mechanical and Aerospace Engineering Department, Carleton University \\ 1125 Colonel By Dr, Ottawa, Canada \\ kyle.chisholm@carleton.ca; mojtaba.ahmadi@carleton.ca
}

\section{Extended Abstract}

Gait rehabilitation robots typically focus on guiding patients through predefined average gait trajectories to facilitate motor learning and have the patients regain their ability to walk through repetitive, high intensity, and cognitively engaging therapy. More recently, virtual environments and visual feedback have been adopted by research platforms with foot-plate based interfaces that can provide force feedback similar to upper-extremity graspable haptic interfaces [1]. The Virtual Gait Rehabilitation Robot (ViGRR) [2], developed at Carleton University's Advanced Biomechatronics and Locomotion Laboratory, is one such device that can administer interactive virtual tasks and gamification of rehabilitation exercise for bedridden patients. In order to ensure the comfort and safety of the user while maintaining a large workspace for various lower-extremity exercises, a force-field safety strategy was developed for the ViGRR platform to prevent hyper extension or flexion. This force-field scheme was implemented in simulation to demonstrate how it can safely allow for a larger workspace compared to fixed Cartesian space or leg joint limits. A calibration methodology was also implemented.

The typical approach to preventing hyper extension or flexion is to inhibit leg joint angles based on predefined user limits or to have detachable interfaces and harness systems in case of falls [3]. However, this approach is problematic due to passive restoring leg joint torques caused by proprioceptive responses that protect muscles and tendons in the leg from injury, limit the range of motion in each joint, and are dependent on other joint angles in the leg [4]. For example, a selected minimum knee joint flexion of 20 degrees while the hip is flexed would inhibit standing and render a sit-to-stand exercise task impossible to perform. Alternatively, constraining each task to a time-varying trajectory violates the desired neurorehabilitation therapy paradigm where the patient should be self-initiating movements, in charge, and engaged.

The proposed solution is to mimic the biomechanics of passive elastic joint torques in the leg. A new force-field function that amplifies a restoring leg torque model mapped to the end effector is introduced. Two methods of calibrating the leg models are applied: the first involves a position-controlled trajectory of the leg and a non-linear optimization of the passive leg joint torque parameters; the second method involves measuring self-reported static joint limits of the user before donning the robot in order to fully characterize exponential restoring joint torque curves. The safety force-field was tested in simulation with an anthropometric leg model falling while interacting with ViGRR and a virtual environment. Preliminary results show that the presented force-field safety concept is viable for further study with ViGRR and may be adopted by other platforms such as exoskeletons for safer physical human-robot integration.

\section{References}

[1] L. Marchal-Crespo and D. J. Reinkensmeyer, "Review of control strategies for robotic movement training after neurologic injury," J. Neuroeng. Rehabil., vol. 6, p. 20, 2009.

[2] K. Chisholm, K. Klumper, A. Mullins, and M. Ahmadi, "A task oriented haptic gait rehabilitation robot," Mechatronics, vol. 24, no. 8, pp. 1083-1091, 2014.

[3] H. Schmidt, S. Hesse, and R. Bernhardt, "Safety concept for robotic gait trainers," in Proc. 26th Annual International Conference of the IEEE Engineering in Medicine and Biology Society, 2004, vol. 1, pp. 2703-2706.

[4] R. Riener and T. Edrich, "Identification of passive elastic joint moments in the lower extremities." J. Biomech., vol. 32, no. 5, pp. 539-544, 1999. 